the glucocorticoid part of the HPA axis, measured through CAR, is involved in the causal pathway between occupational and environmental noise exposure and cardiovascular disease.

\section{EXPOSURE TO PHTHALATES, PERFLUORINATED COMPOUNDS AND ORGANOCHLORINES AND PREGNANCY OUTCOMES IN WOMEN FROM GREENLAND, POLAND AND UKRAINE}

${ }^{1} V$ C Lenters, ${ }^{1}$ Portengen, ${ }^{2}$ Rignell-Hydbom, ${ }^{2}$ Jönsson, ${ }^{2}$ Lindh, ${ }^{3}$ Ludwicki, ${ }^{4}$ Pedersen, ${ }^{5}$ Zviezdai, ${ }^{6}$ Piersma, ${ }^{7}$ Toft, ${ }^{8}$ Bonde, ${ }^{1}$ Heederik, ${ }^{2}$ Rylander, ${ }^{1}$ Vermeulen. ${ }^{1}$ Utrecht University, Utrecht, Nederland; ${ }^{2}$ Lund University, Lund, Sweden; ${ }^{3}$ National Institute of Public HealthNational Institute of Hygiene, Warsaw, Poland; ${ }^{4}$ Centre for Arctic Environmental Medicine, Nuuk, Greenland; ${ }^{5}$ Kharkiv National Medical University, Kharkiv, Ukraine; ${ }^{6}$ National Institute for Public Health and the Environment (R/VM), Bilthoven, The Netherlands; 'Aarhus University, Aarhus, Denmark; ${ }^{8}$ Copenhagen University Hospital, Copenhagen, Denmark

\subsection{6/oemed-2013-101717.399}

Objectives Evidence for effects of environmental contaminants on pregnancy outcomes remains inconclusive. We investigated the associations between multiple, correlated exposures and related pregnancy outcomes using Bayesian, multivariate dimension reduction, and shrinkage regression approaches to account for multiple testing and interrelatedness of exposures and outcomes.

Methods We evaluated a cohort of 1322 singletons, born to 547 mothers from Greenland, 197 from Warsaw, Poland, and 588 from Kharkiv, Ukraine, who were recruited in 2002-2004 during routine antenatal care visits. Three secondary metabolites of both diethylhexyl and diisononyl phthalates (DEHP, DINP), eight perfluorinated compounds (PFCs; including PFOS and PFOA), and organochlorines ( $p, p$-DDE and PCB-153) were measured and detected in $72-100 \%$ of maternal serum samples. Outcomes were preterm birth $(<37$ weeks), birth weight, and small for gestational age (SGA; $<10^{\text {th }}$ percentile age- and gender-specific birth weight). We analysed exposures (clustered, high dimension predictors) and continuous and dichotomous outcomes with partial least squares (PLS) regression, and sparse PLS-discriminant analysis (sPLS-DA), respectively. We compared results with elastic net penalised regression, and Bayesian stochastic search variable selection with spike-and-slab priors of (nonlinear) generalised additive models.

Results While applied methods had various degrees of sparseness, we observed generally consistent associations between DEHP metabolites, several PFCs and both organochlorines, and decreased birth weight and increased risk of SGA. There was no clear evidence of associations between contaminants and preterm birth. Conclusions Findings suggest that several environmental contaminants are independently associated with impaired fetal growth. Methods which account for correlations between variables and multiple testing may better discriminate robust exposure-response associations than conventional univariate linear and logistic regression models.

\section{CIRCULATING SOLUBLE CD27 AND CD30 IN WORKERS EXPOSED TO 2, 3, 7, 8-TETRACHLORODIBENZO-P-DIOXIN (TCDD)}

${ }^{1} \mathrm{~F}$ Saberi Hosnijeh, ${ }^{2}$ Portengen, ${ }^{3}$ Bueno-de-Mesquita, ${ }^{2}$ Heederik, ${ }^{1}$ Vermeulen. ${ }^{1}$ Utrecht University, Utrecht, The Netherlands; ${ }^{2}$ Institute for Risk Assessment Sciences (IRAS), Utrecht University, Utrecht, The Netherlands; ${ }^{3}$ National Institute for Public Health and Environment (RIVM), Bilthoven, The Netherlands

10.1136/oemed-2013-101717.400
Objectives Previous studies suggest that 2, 3, 7, 8-tetrachlorodibenzo-p-dioxin (TCDD) exposure may be associated with nonHodgkin lymphoma (NHL) but findings remain inconclusive. There is a need for mechanistic studies to evaluate the biologic plausibility of this association. In this cross-sectional study we investigated changes in plasma levels of two soluble markers of B cell activation, sCD27 and sCD30 and IL1RA, which have been found to be predictive of lymphoma, among workers from a Dutch historical cohort occupationally exposed to chlorophenoxy herbicides and contaminants including TCDD.

Methods Eighty-five workers who had been exposed to either high $(n=47)$ or low $(n=38)$ TCDD levels more than 30 years before serum collection were included in the current investigation. Plasma level of the sCD27, sCD30, and IL1RA was measured by ELISA. Current plasma levels of TCDD (TCDD Current $_{\text {) }}$ ) were determined by high-resolution gas chromatography/isotope dilution high resolution mass spectrometry. TCDD blood levels at the time of last exposure $\left(\mathrm{TCDD}_{\max }\right)$ were estimated using a one-compartment first order kinetic model.

Results Dose-response analyses showed no significant association between blood levels of sCD27, sCD30 and IL1RA and current and estimated past maximum TCDD levels although there was an indication of decreased levels of all markers with increasing TCDD level. Analyses excluding subjects with chronic diseases resulted in a significant decrease in IL1RA with increasing levels of TCDD.

Conclusions No significant dose-response relationship was observed between the measured markers and TCDD level in our study. However, there was a suggestion that sCD27, sCD30 and IL1-RA tended to decrease with increasing TCDD levels. This later observation is consistent with the earlier observation on decreasing cytokine levels with increasing exposures.

\section{Session: 34. Health impact analysis II}

\section{COST-BENEFIT ANALYSIS OF INTERVENTIONS USING A PROBABILISTIC APPROACH: A CASE FOCUSING ON QUARTZ EXPOSURE IN THE DUTCH CONSTRUCTION INDUSTRY}

B van Duuren, Meijster, Vergeer, de Weerd. TNO, Zeist, Nederland

10.1136/oemed-2013-101717.401

Objectives Ill-health due to exposure in the workplace results in high costs for employers, employees and society. Interventions can be costly and economic evaluations receive more and more attention in the decision making processes regarding investments, including for occupational health. To perform an economic evaluation information regarding the impact of interventions on exposure and subsequently, health and work performance is needed. Additionally, information regarding costs is needed. Meijster et al. (2011) presented an approach to evaluate the costs and benefits for different stakeholders. We further developed this approach into a probabilistic model to include variability for input parameters and obtain uncertainty estimates for output parameters. This approach is applied to a hypothetical case study focusing on reducing quartz exposure in the Dutch construction industry.

Methods The original cost-benefit approach was further developed into a probabilistic approach including Monte Carlo simulations using Excel spreadsheets. This enables the user to calculate total costs, total benefits, net costs and cost-effectiveness which can be easily applied for different intervention (s). 